\title{
Prolungamenti finiti di un corpo ed algebre gruppali $\left(^{(*)}\left({ }^{* *}\right)\right.$.
}

\author{
Marto Poletiti (Pisa)
}

Sunto. - Per ciascun prolungamento finito di un corpo di caratteristica positiva, viene introdotto un T-modulo canonico; si determina la struttura dei moduli degli omomorfismi tra questi. In tale contesto, varie algebre gruppali ricevono interpretazioni canoniche in termini di teoria di Galois. Si dànno teoniche per tradurre, ciascuno nel linguaggio degli altri, problemi di teoria di Galois, di T-moduli canonici, di algebre gruppali.

In [1], per ciascun prolungamento finito $F$ di un corpo $k$ di caratteristica positiva, viene definita un'iperalgebra locale $R_{F}$ su $k$, le cui principali proprietà ivi indicate sono: i) $F$, come prolungamento di $k$, è quoziente dell'algebra delle iperderivazioni 1-speciali invarianti di $R_{p}$; ii) il $T_{k}$-modulo canonico $\cup D_{F}$ dei vettori canonici di Witt dell'iperalgebra $D_{F}$ duale di $R_{F}$, è isomorfo al $T_{k}$-modulo canonico vect $F$; iii) prolungamenti di $k$ aventi iperalgebre associate isomorfe, hanno ehiusure normali su $k$ isomorfe; iv) prolungamenti normali di $k$ aventi iperalgebre associate isomorfe, sono isomorfi. Infine in [1] exa emerso che tra due prolungamenti di $k$ aventi iperalgebre associate isomorfe, esiste un isomorfismo di spazi vettoriali su $k$, commutante con i rispettivi endomorfismi di Frobenius.

Il presente lavoro prende lo spunto da tale ultima considerazione. Definiti i $\pi$-omomorfismi di un prolungamento finito di $k$, se ne determina l'insieme, e (nel caso separabile) si caratterizzano quelli aventi immagine in un prefissato prolungamento di $k$. Parallelamente, al fine di collegare il $\pi$-isomorfismo tra prolungamenti, all'isomorfismo delle iperalgebre associate, vengono studiati gli omomorfismi tra i $T_{k}$-moduli canonici dei vettori di Witt a componenti nei prolungamenti finiti di $k$. Il risultato più significativo al riguardo è che ciascun $\pi$-isomorfismo tra prolungamenti separabili di $k$, si rialza ad un isomorfismo dei $T_{k}$-moduli canonici dei relativi vettori di Witt, e conseguentemente induce un isomorfismo tra le iperalgebre associate.

Le tecniche usate portano ad attribuire, in termini di teoria di Galois, significati naturali alle algebre gruppali di un gruppo finito, sul corpo fondamentale di caratteristica $p$, sull'anello degli interi $p$-adici, sul corpo dei razionali $p$-adici.

In tale contesto, la congettura posta in [1], che prolungamenti di $k$ aventi iperalgebre associate isomorfe siano isomorfi, trova, nel caso separabile, formulazioni

(*) Entrata in Redazione il 14 ottobre 1976.

(**) Lavoro eseguito nell'ambito del Gruppo Nazionale per le Strutture Algebriche e Geometriche o loro Applicazioni, del C.N.R.

25 - Annali di Matematica 
equivalenti particolarmente semplici. Sulla base di quelle, risulta agevole provare vera tale congettura: $i$ ) per prolungamenti separabili di $k$ aventi ordine primo nella propria chiusura normale; ii) per prolungamenti separabili di $k$ aventi come ordine nella propria chinsura normale, una potenza della loro caratteristica.

In quanto segue, $p$ è un primo positivo, $k$ è un corpo infinito di caratteristica $p$, $C$ è la chiusura algebrica di $k, \pi$ è l'endomorfismo di Frobenius di $C$. Tutti i prolungamenti algebrici di $k$ si intendono immersi in $C$.

Se $F$ è un corpo di caratteristica $p$, indicheremo con vect $F$ l'anello dei vettori infiniti di Witt a componenti in $F$; se $F$ è perfetto, indicheremo con biv $F$ il corpo dei bivettori di Witt a componenti in $F$ (cir. [MA], cap. 2, e [2]). $t$ denota nel primo caso l'operatore $\boldsymbol{t}\left(a_{0}, a_{1}, \ldots\right)=\left(0, a_{0}, a_{1}, \ldots\right)$, e nel secondo l'operatore $\boldsymbol{t}\left(\ldots, a_{-1}\right.$; $\left.a_{0}, \ldots\right)=\left(\ldots a_{-2} ; a_{-1}, a_{0}, \ldots\right)$.

Introdotta un'indeterminata $t$, indicheremo con $T_{k}$ l'anello ottenuto considerando il (vect $k$ )-modulo destro delle serie formali nelle potenze non negative di $t$ a coefficienti (a destra) in vect $k$, e ponendo $a t=t a^{\pi}$ ( $\pi$ è l'endomorfismo di Frobenius di vect $k$ ). Se $k$ è perfetto, indicheremo con $T_{k}^{*}$ l'analogo di $T_{k}$, per il quale si ammettono serie formali in $t$ ad esponenti anche negativi, con la convenzione che in ogni elemento di $T_{k}^{*}$ intervenga solo un numero finito di potenze negative di $t$ (cir. 1, pg. 308 di $[\mathrm{MC}]$ ).

$\boldsymbol{F}_{p}$ è il corpo fondamentale di caratteristica $p ; \boldsymbol{Z}_{p}=\operatorname{vect} \boldsymbol{F}_{p}$ e $\boldsymbol{Q}_{p}=\operatorname{biv} \boldsymbol{F}_{p}$ sono rispettivamente l'anello degli interi $p$-adici ed il corpo dei razionali $p$-adici.

Se $A$ è un campo di integrità, e $G$ è un gruppo, l'algebra gruppale di $G$ su $A$ si indicherà con $A[\mathrm{G}]$.

Isomorfismo significa omomorfismo iniettivo.

1. - Siano $F$, $H$ prolungamenti algebrici di $k$; una applicazione $k$-lineare $f: F \rightarrow H$ commutante con $\pi$, ossia tale che $f\left(a^{D}\right)=f(\alpha)^{p}$ per ogni $a \in F$, si dirà un $\pi$-omomorfismo di $F$ in $H$ su $k$.

L'insieme dei $\pi$-omomorfismi di $F$ in $H$ su $k$, è ovviamente uno spazio vettoriale su $\boldsymbol{F}_{p} ;$ lo denoteremo con $\Pi_{l i}(F, H)$.

1.1. TEOREMA. - Sia $F$ un protungamento finito di $k$, e siano $\sigma_{1}, \ldots, \sigma_{s}$ gli isomorfismi di $F$ in $O$ sit $l$.

$\sigma_{1}, \ldots, \sigma_{s} \grave{e}$ una base di $\Pi_{k}(F, C)$ come spazio vettoriale su $\boldsymbol{F}_{p}$.

Drv. - Che $\sigma_{1}, \ldots, \sigma_{s}$ siano elementi di $\Pi_{k}(F, C)$ è ovvio; che siano linearmente indipendenti su $C$ e quindi su $\boldsymbol{F}_{p}$ è ben noto (cfr. [3], pg. 25).

Siano quindi $g_{1}, \ldots, g_{t}\left(t=p^{s}\right)$ gli elementi del sottospazio vettoriale di $\Pi_{k}(F, C)$ generato da $\sigma_{1}, \ldots, \sigma_{s}$, e sia $f \in \Pi_{k}(F, C)$.

Sia $a$ un elemento qualsiasi di $\not F$. Detto $I$ l'ideale di $k[X]$ generato dai polinomi del tipo

$$
a_{0} X^{p^{0}}+a_{1} X^{p^{1}}+\cdots+a_{r} X^{p^{r}} \in k[X]
$$


aventi $a$ per radice, si constata che $f(a)$ è radice di ciascuno di tali polinomi, ed è pertanto radice del generatore $\mu(X)$ di $I$. Le radici di $\mu(X)$ sono (ofr. la dimostrazione di 2.1 di [1]) gli elementi dello spazio vettoriale generato su $\boldsymbol{F}_{p}$ dai coniugati di $a$ su $k$. Esiste quindi $i$, a priori dipendente da $a$, tale che $f(a)=g_{i}(a)$, ossia tale che $a \in K_{i}=\operatorname{ker}\left(f-g_{i}\right)$. Ne segue $F=K_{1} \cup \ldots \cup K_{t}$.

Sia $\theta_{1}, \ldots, \theta_{n}$ una base di $F$ come spazio vettoriale su $k$, e sia o la dualità tra $F$ e se stesso (su $k$ ) definita da $\theta_{i} \circ \theta_{j}=\delta_{i j}$. Se per $i=1, \ldots, t$ fosse $K_{i} \neq F$, allora (immergendo $K_{i}$ in un sottospazio di $F$ di dimensione $n-1$, ossia nell'ortogonale di un elemento di $F$ ) per ciascun tale $i$ esisterebbe $\eta_{i} \neq 0$ tale che $\eta_{i} \circ K_{i}=\{0\}$. Posto quindi $\eta_{i}=\sum_{\lambda=1}^{n} a_{i \lambda} \theta_{\lambda}\left(a_{i \lambda} \in k\right)$, dati comunque $x_{1}, \ldots, x_{n} \in k$ e posto $\theta=\sum_{\lambda=1}^{n} x_{\lambda} \theta_{\lambda}$, essendo $F=K_{1} \cup \ldots \cup K_{t}$, si avrebbe

$$
\left(\theta \circ \eta_{1}\right) \ldots . .\left(\theta \circ \eta_{t}\right)=\left(\sum_{\lambda=1}^{n} a_{1 \lambda} x_{\lambda}\right) \ldots . .\left(\sum_{\lambda=1}^{n} a_{t \lambda} x_{\lambda}\right)=0 .
$$

Essendo $k$ infinito, il polinomio

$$
\left(\sum_{\lambda=1}^{n} a_{1 \lambda} X_{\lambda}\right) \ldots\left(\sum_{\lambda=1}^{n} a_{t \lambda} X_{\lambda}\right) \in k\left[X_{1}, \ldots, X_{n}\right]
$$

risulterebbe nullo, e pertanto risulterebbe nullo un suo fattore.

Esisterebbe quindi $j$ tale che $a_{j 1}=\ldots=a_{i n}=0$, ossia tale che $\eta_{j}=0$ : assurdo.

Si conclude quindi che esiste $i$ tale che $K_{i}=F$, ossia tale che $\operatorname{ker}\left(f-g_{i}\right)=F$; per tale $i$ si ha $f=g_{i}$, C.V.D.

Sia $N$ un qualsiasi prolungamento di $F$, normale su $k$; tenuto eonto di 1.1 si ha:

1.2. $\Pi_{k}(F, C)=\Pi_{k}(F, N)$;

1.3. l'applicazione di $\Pi_{k}(N, N)$ in $\Pi_{k}(F, N)$ che a ciasoun elemento di $\Pi_{k}(N, N)$ associa la sua restrizione ad $F$, ̀̀ surgettiva.

Siano $F, H$ prolungamenti algebrici di $k$; vect $F$ e veet $H$ risultano $T_{k}$-moduli; se poi $F$ ed $H$ sono finiti su $k$, vect $F$ e vect $H$ risultano canonici (cfr. 4 di [1]). L'insieme degli omomorfismi di veet $F$ in veet $H$ come $T_{k}$-moduli, è ovviamente un modulo su $\boldsymbol{Z}_{p} ;$ lo denoteremo con $\Pi_{k}$ (vect $F$, vect $H$ ).

1.4. Teorema. - Sia $F$ un prolungamento finito $d i k$, siano $\sigma_{1}, \ldots, \sigma_{s}$ gli isomorfismi di $F$ in $O$ su $k$, e siano $\tilde{\sigma}_{1}, \ldots, \tilde{\sigma}_{s}$ gli isomorfismi di vect $F$ in vect $C$ definiti da: $\tilde{\sigma}_{i}\left(a_{0}, a_{1}, \ldots\right)=\left(\sigma_{i} a_{0}, \sigma_{i} a_{1}, \ldots\right)$.

$\tilde{\sigma}_{1}, \ldots, \tilde{\sigma}_{s} \grave{e}$ una base di $\Pi_{k}$ (veet $F$, vect $C$ ) come modulo su $\boldsymbol{Z}_{p}$.

DrM. - Ohe $\tilde{\sigma}_{1}, \ldots, \tilde{\sigma}_{s}$ siano elementi di $\Pi_{k}($ vect $F$, veot $C$ ), è subito visto. Siano quindi $c_{1}=\left(c_{10}, c_{11}, \ldots\right), \ldots, c_{s}=\left(c_{s 0}, c_{s 1}, \ldots\right)$ elementi di $\boldsymbol{Z}_{p}$ tali che $c_{1} \tilde{\sigma}_{1}+\ldots+c_{s} \tilde{\sigma}_{s}=0$. Il $\pi$-omomorfismo $c_{10} \sigma_{1}+\ldots+c_{s 0} \sigma_{s}$ di $F$ in $C$, ̀̀ nullo, pertanto $c_{10}=\ldots=c_{30}=0$. 
Ne segue $p\left[\left(c_{11}, c_{12}, \ldots\right) \tilde{\sigma}_{1}+\ldots+\left(c_{s 1}, c_{s 2}, \ldots\right) \tilde{\sigma}_{s}\right]=0$, da cui $\left(c_{11}, c_{12}, \ldots\right) \tilde{\sigma}_{1}+\ldots+$ $+\left(e_{s 1}, e_{s 2}, \ldots\right) \tilde{\sigma}_{s}=0$; se ne deduce $e_{11}=\ldots=e_{s 1}=0$. Così proseguendo si prova che $c_{1}=\ldots=\boldsymbol{c}_{s}=0$, ossia che $\tilde{\sigma}_{1}, \ldots, \tilde{\sigma}_{s}$ sono indipendenti su $\boldsymbol{Z}_{p}$.

Si consideri adesso un omomorfismo $f:$ vect $F \rightarrow$ vect $C$, come $T_{k 0}$-moduli; sia $f^{\prime}:\left(\right.$ vect $\left.F^{\prime}\right) / t($ vect $F) \rightarrow($ vect $C) / t($ vect $C)$ l'applicazione indotta da $f$. Identificando (vect $F) / t($ vect $F)$ con $F$, e (vect $C) / t($ vect $C)$ con $C$, si constata che $f^{\prime} \in \Pi_{k}(F, C)$; per 1.1 esistono allora $e_{10}, \ldots, c_{s 0} \in \boldsymbol{F}_{p}$ tali che $f^{\prime}=c_{10} \sigma_{1}+\ldots+c_{s 0} \sigma_{s}$. Pertanto, posto $f_{1}=f-\left[\left\{c_{10}\right\} \tilde{\sigma}_{1}+\ldots+\left\{c_{s 0}\right\} \tilde{\sigma}_{s}\right]$ (ove $\{c\}=(c, 0,0, \ldots)$ ), si ha: $\operatorname{Im} f_{1} \subseteq t($ vect $\sigma)$.

Procedendo per induzione, supponiamo determinati, per $j=1, \ldots, m$, elementi $e_{1 i}, \ldots, c_{s j} \in \boldsymbol{F}_{p}$ tali che, posto

$$
\begin{aligned}
f_{j}=f-\left[\left(\left\{c_{10}\right\}+p^{1}\left\{c_{11}\right\}+\ldots+p^{j-1}\left\{c_{1, j-1}\right\}\right)\right. & \tilde{\sigma}_{1}+\ldots+ \\
& \left.+\left(\left\{c_{s 0}\right\}+p^{\mathbf{1}}\left\{c_{s 1}\right\}+\ldots+p^{j-1}\left\{c_{s, j-1}\right\}\right) \tilde{\sigma}_{s}\right],
\end{aligned}
$$

si abbia: $\operatorname{Im} f_{j} \subseteq t^{j}($ vect $C)$.

Si consideri quindi $f_{m}$ : veet $F \rightarrow$ vect $C$; essendo $\operatorname{Im} f_{m} \subseteq t^{m}($ vect $C)$, esiste una ed una sola applicazione $\omega$ : vect $F \rightarrow$ vect $C$, tale che $f_{m}=\boldsymbol{t}^{m} \omega$. Si constata facilmente che: i) $\omega$ è un omomorfismo additivo, ii) $\omega t=t \omega$, iii) $\omega \pi=\pi \omega$, iv) se $c \in k$ ed $a \in$ vect $F$, si ha $\omega(\{0\} a)=\left\{c^{p^{m}}\right\} \omega(a)$. Tenuto conto di i $)$, ii), previa 1'identificazione detta precedentemente, $\omega$ induce un omomorfismo additivo $\omega^{\prime}: F \rightarrow C$, verificante le proprietà: $\left.\operatorname{iii}^{\prime}\right) \omega^{\prime}\left(a^{p}\right)=\omega^{\prime}(a)^{p}$, i $\left.\nabla^{\prime}\right)$ se $c \in k$ ed $a \in F$, si ha $\omega^{\prime}(c a)=$ $=e^{p^{m}} \omega^{\prime}(a)$. Detta quindi $\omega^{*}: F^{\prime} \rightarrow C$ l'applicazione definita da $\omega^{*}(a)=\left(\omega^{\prime}(a)\right)^{1 / p^{m}}$, si constata che $\omega^{*} \in \Pi_{k}(F, C)$; per 1.1 esistono allora $c_{1 m}, \ldots, c_{s m} \in \boldsymbol{F}_{p}$, tali che $\omega^{*}=c_{1 m} \sigma_{1}+\ldots+c_{s m} \sigma_{s}$.

Si ha allora $\omega^{\prime}=\pi^{m}\left(e_{1 m} \sigma_{1}+\ldots+c_{s m} \sigma_{s}\right)$, pertanto per ogni $a \in$ vect $F$ risulta

$$
\omega(a) \equiv \pi^{m}\left(\left\{c_{1 m}\right\} \tilde{\sigma}_{\perp}+\ldots+\left\{c_{s m}\right\} \tilde{\sigma}_{s}\right)(a) \quad \bmod t(\text { vect } C)
$$

quindi

$$
t^{m} \omega(a) \equiv t^{m} \pi^{m}\left(\left\{c_{1, m}\right\} \tilde{\sigma}_{1}+\ldots+\left\{c_{s m}\right\} \tilde{\sigma}_{s}\right)(a) \quad \bmod t^{m+1}(\text { vect } O)
$$

ossia

$$
f_{m}(a) \equiv\left(p^{m}\left\{c_{1 m}\right\} \tilde{\sigma}_{1}+\cdots+p^{m}\left\{c_{s m}\right\} \tilde{\sigma}_{s}\right)(a) \quad \bmod t^{m+1}(\text { vect } C) .
$$

Posto infine

$$
\begin{aligned}
f_{m+1}=f_{m}-\left(p^{m}\left\{c_{1 m}\right\} \tilde{\sigma}_{1}+\ldots\right. & \left.+p^{m}\left\{c_{s m}\right\} \tilde{\sigma}_{s}\right)= \\
=f-\left[\left(\left\{c_{10}\right\}+p^{1}\left\{c_{11}\right\}\right.\right. & \left.+\ldots+p^{m}\left\{c_{1 m}\right\}\right) \tilde{\sigma}_{1}+\ldots+ \\
& \left.+\left(\left\{c_{s 0}\right\}+p^{1}\left\{c_{s 1}\right\}+\ldots+p^{m}\left\{c_{s m}\right\}\right) \tilde{\sigma}_{s}\right],
\end{aligned}
$$

si ha: $\operatorname{Im} f_{m+1} \subseteq t^{m+1}($ vect $C)$.

Posto, per $i=1, \ldots, s, c_{i}=\left(c_{i 0}, c_{i 1}, \ldots\right) \in \boldsymbol{Z}_{p}$, ove i $c_{i j}$ sono gli elementi di $\boldsymbol{F}_{p}$ definiti col procedimento induttivo descritto, si ha: $f=c_{1} \tilde{\sigma}_{1}+\ldots+c_{s} \tilde{\sigma}_{s}, \quad$ C.V.D. 
Sia $N$ un qualsiasi prolungamento di $F$, normale su $k$; tenuto conto di 1.4 si ha:

1.5. $\Pi_{k}($ vect $F$, vect $C)=\Pi_{k}($ vect $F$, vect $N)$;

1.6. l'copplicazione di $\Pi_{k}$ (vect $N$, vect $\left.N\right)$ in $\Pi_{k}($ vect $F$, vect $N)$ che a ciascun elemento di $\Pi_{k}($ veet $N$, vect $N)$ associa la sua restrizione a vect $F$, è surgettiva.

Tenuto infine conto di 1.1 e di 1.4 si ha:

1.7. Vapplicazione $d i \Pi_{k}($ vect $F$, vect $N)$ in $\Pi_{\bar{k}}(F, N)$, che a ciascun elemento $f \in \Pi_{k}\left(\right.$ vect $\not F$, vect $N$ ) associa l'elemento $f^{\prime} \in \Pi_{k}(F, N)$ indotto da $f$ (previa identificazione $d i($ vect $F) / t($ vect $F)$ con $F$, e di $($ vect $N) / t($ vect $N)$ con $N)$, ̀̀ surgettiva.

2. - Sia $N$ un prolungamento di Galois (ossia finito, separabile e normale) di $k$, e ne sia $G=\mathscr{G}(N / k)$ il gruppo di Galois.

2.1. Teonema. - L'algebra gruppale $\boldsymbol{F}_{p}[G]$ è canonioamente isomorfa alla $F_{p^{-}}$-algebra dei $\pi$-endomorfismi di $N$ su $k$.

DIM. - L'esistenza di un omomorfismo canonico della prima nella seconda delle algebre dette, è banale; che tale omomorfismo risulti un isomorfismo surgettivo, segue da 1.1, C.V.D.

2.2. TEOREMA. - L'algebra gruppale $\boldsymbol{Z}_{p}[G]$ è canonicamente isomorfa alla $\boldsymbol{Z}_{p}$-algebra degli endomorfismi di vect $N$ come $T_{k}$-modulo canonico.

DrM. - L'applicazione che a ciaseun elemento $\sigma \in G$ fa corrispondere l'elemento $\tilde{\sigma}$ definito da $\tilde{\sigma}\left(a_{0}, a_{1}, \ldots\right)=\left(\sigma a_{0}, \sigma a_{1}, \ldots\right)$, si prolunga canonicamente ad un omomorfismo della prima nella seconda delle algebre dette; che tale omomorfismo risulti un isomorfismo surgettivo, segue da 1.4, C.V.D.

Se $k$ è perfetto, si considerino i corpi biv $k$, biv $N$; biv $N$ risulta essere un $T_{k}^{*}$-modulo finitamente generato.

Detti $\sigma_{1}, \ldots, \sigma_{n}$ gli elementi di $G$, e posto $\tilde{\sigma}_{i}\left(\ldots, a_{-1} ; a_{0}, a_{1}, \ldots\right)=\left(\ldots, \sigma_{i} a_{-1}\right.$; $\left.\sigma_{i} a_{0}, \sigma_{i} a_{1}, \ldots\right)$, l'insieme $\widetilde{G}=\left\{\tilde{\sigma}_{1}, \ldots, \tilde{\sigma}_{n}\right\}$ rișulta essere un gruppo di automorfismi del corpo biv $N$, gruppo ovviamente isomorfo a $G$. Il sottocorpo costituito dagli elementi di biv $N$ stabili rispetto a $\tilde{G}$, è ovviamente biv $k$; in particolare, biv $N$ è un prolungamento di Galois di biv $k$, e si ha $\mathbf{S}($ biv $N /$ biv $k)=\widetilde{G}$.

2.3. TEOREMA. - L'algebra gruppale $\boldsymbol{Q}_{\mathcal{p}}[G]$ è canonicamente isomorfa alla $\boldsymbol{Q}_{p}$-algebra degli endomorfismi di biv $N$ come $T_{k}^{*}$-modulo.

Drm. - L'applicazione che a ciascun $\sigma_{i}$ fa corrispondere $\tilde{\sigma}_{i}$, si prolunga canonicamente ad un omomorfismo della prima nella seconda delle algebre dette.

Siano $c_{1}, \ldots, c_{n} \in \boldsymbol{Q}_{p}$ tali che $c_{1} \tilde{\sigma}_{1}+\ldots+\boldsymbol{c}_{n} \widetilde{\sigma}_{n}=0$; scelto $h$ tale che ogni $p^{h} c_{i} \in \boldsymbol{Z}_{p}$, l'elemento $\left(p^{h} c_{1}\right) \tilde{\sigma}_{1}+\ldots+\left(p^{h} c_{n}\right) \tilde{\sigma}_{n}$, ristretto a vect $N$, risulta l'endomorfismo nullo 
di vect $N$; tenuto conto di 2.2 si ha $p^{h} c_{1}=\ldots=p^{h} c_{n}=0$, ossia $c_{1}=\ldots=c_{n}=0$. Ne segue che l'omomorfismo detto è un isomorfismo; che risulti surgettivo si prova come segue.

Sia $f:$ biv $N \rightarrow$ biv $N$ una applicazione $T_{k}^{*}$-lineare; detto $x_{1}, \ldots, x_{n}$ un sistema di generatori di vect $N$ come $T_{k^{-}}$-modulo canonico, esiste $h$ tale che $p^{h} f\left(x_{1}\right), \ldots, p^{h} f\left(x_{n}\right) \in$ $\in$ vect $N$; conseguentemente $p^{n} f($ vect $N) \subseteq$ veet $N$. Per 2.2 esistono allora $c_{1}, \ldots, c_{n} \in Z_{p}$ tali che per ogni $x \in$ vect $N$ si abbia $p^{n} f(x)=c_{1} \tilde{\sigma}_{1}(x)+\ldots+c_{n} \tilde{\sigma}_{n}(x)$, ossia $f(x)=$ $=\left(p^{-h} c_{1}\right) \tilde{\sigma}_{1}(x)+\ldots+\left(p^{-h} c_{n}\right) \tilde{\sigma}_{n}(x)$; che tale uguaglianza sussista per ogni $x \in$ biv $N$, si constata tenendo conto che, se $x \in b i v N$, allora $p^{i} x \in$ vect $N$ per $l$ opportuno,

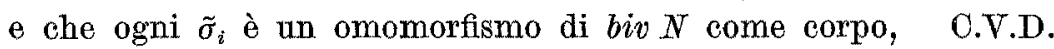

3. - Siano $F, H$ prolungamenti finiti e separabili di $k$, e sia $N$ un prolungamento di Galois di $k$, contenente $F$ ed $H$.

Si ponga $G=\mathfrak{G}(N / k)$, $\mathscr{F}=\mathfrak{G}(N / F)$, $\mathfrak{H}=\mathfrak{S}(N / H)$.

Siano $\sigma_{1} \mathscr{F}, \ldots, \sigma_{s} \mathscr{F}$ le classi laterali sinistre di $\mathscr{F}$ in $G$, così che le restrizioni di $\sigma_{1}, \ldots, \sigma_{s}$ ad $F$ (che in quanto segue indicheremo con gli stessi simboli $\sigma_{1}, \ldots, \sigma_{s}$ ) risultano gli isomorfismi di $F$ in $N$, su $k$.

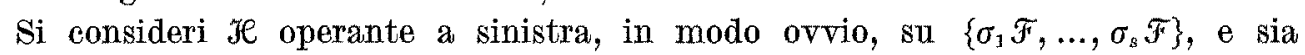
$\left\{\sigma_{11} \mathfrak{F}, \ldots, \sigma_{1 s_{1}} \mathfrak{F}\right\}, \ldots,\left\{\sigma_{t 1} \mathfrak{F}, \ldots, \sigma_{t \varepsilon_{t}} \mathscr{F}\right\}$ (con ogni $\sigma_{i j}$ scelto tra $\left.\sigma_{1}, \ldots, \sigma_{s}\right)$ la conseguente partizione in orbite di $\left\{\sigma_{1} \mathscr{F}, \ldots, \sigma_{s} \mathscr{F}\right\}$.

3.1. Lemma. - Gli elementi $\eta_{1}=\sigma_{11}+\ldots+\sigma_{1 s_{1}}, \ldots, \eta_{t}=\sigma_{t 1}+\ldots+\sigma_{t s_{t}} d i$ $\Pi_{k}(\boldsymbol{F}, N)$, sono una base di $\Pi_{k}(\boldsymbol{F}, \boldsymbol{H})$ come modulo su $\boldsymbol{F}_{p}$.

Dim. - Sia $a \in F$; per come scelti $\sigma_{i 1}, \ldots, \sigma_{i s_{i}}$, dato comunque $\varrho \in \mathfrak{S}(N / H)$, essendo $a \in F$, si ha $\varrho \eta_{i}(a)=\eta_{i}(a)$, pertanto $\eta_{i}(a) \in H$; ne segue che $\eta_{1}, \ldots, \eta_{t} \in \Pi_{k}(F, H)$.

L'indipendenza di $\eta_{1}, \ldots, \eta_{t}$ su $\boldsymbol{F}_{p}$, tenuto conto che $\left\{\sigma_{11}, \ldots, \sigma_{1 s_{1}}\right\}, \ldots,\left\{\sigma_{t 1}, \ldots, \sigma_{t s_{t}}\right\}$ è una partizione di $\left\{\sigma_{1}, \ldots, \sigma_{s}\right\}$, segue da 1.1 .

Si consideri quindi un elemento $f \in \Pi_{k}(F, H)$. Essendo $\Pi_{k}(F, H) \subseteq \Pi_{k}(F, N)$, per 1.1 esistono e sono univocamente determinati elementi $a_{1}, \ldots, a_{s} \in \boldsymbol{F}_{p}$ tali che $f=a_{1} \sigma_{1}+\ldots+a_{s} \sigma_{s}$; se $\sigma_{i j}=\sigma_{r}$, si ponga $a_{i j}=a_{r}$. Fissato $i=1, \ldots, t$, e scelti $j, h$ compresi tra 1 ed $s_{i}$, esiste $\varrho \in \mathcal{G}(N / H)$ tale che $\varrho \sigma_{i j} \mathfrak{F}=\sigma_{i \hbar} \mathfrak{F}$; considerando $\sigma_{1}, \ldots, \sigma_{s}$ come isomorfismi di $F$ in $N$, su $k$, si ha che $\varrho \sigma_{1}, \ldots, \varrho \sigma_{s}$ è una permutazione di $\sigma_{1}, \ldots, \sigma_{s}$, e che $\varrho \sigma_{i j}=\sigma_{i \hbar} ;$ pertanto, essendo $f=a_{1} \sigma_{1}+\ldots+a_{s} \sigma_{s}, f=\varrho f=$ $=a_{1} \varrho \sigma_{1}+\ldots+a_{s} \varrho \sigma_{s}$, si ha $a_{i \hbar}=a_{i j}$. Ne segue: $f=a_{11}\left(\sigma_{11}+\ldots+\sigma_{1 s_{1}}\right)+\ldots+$ $+a_{t s}\left(\sigma_{t 1}+\ldots+\sigma_{t s_{t}}\right), \quad$ C.V.D.

3.2. LEMMA. - Gli elementi (cfr. 1.4) $\xi_{1}=\tilde{\sigma}_{11}+\ldots+\tilde{\sigma}_{1 s_{1}}, \ldots, \xi_{t}=\tilde{\sigma}_{t 1}+\ldots+\tilde{\sigma}_{t s_{t}}$ di $\Pi_{k}($ vect $F$, vect $N)$, sono una base $d i \Pi_{k i}$ (vect $F$, vect $H$ ) come modulo su $Z_{p}$.

DIM. - Sia $a \in$ vect $F$; per come scelti $\sigma_{i 1}, \ldots, \sigma_{i s_{i}}$, dato comunque $\varrho \in \mathbb{G}(N / H)$, essendo ogni componente di $a$ elemento di $F$, si ha che $\tilde{\varrho} \tilde{\sigma}_{i 1}(a), \ldots, \tilde{\varrho} \tilde{\sigma}_{i s_{i}}(a)$ è una permutazione di $\tilde{\sigma}_{i 1}(a), \ldots, \tilde{\sigma}_{i s_{i}}(a)$; conseguentemente $\tilde{\varrho}\left(\tilde{\sigma}_{i 1}(a)+\ldots+\tilde{\sigma}_{i s_{i}}(a)\right)=\tilde{\sigma}_{i 1}(a)+$ 
$+\ldots+\tilde{\sigma}_{i s_{i}}(a)$, e pertanto ogni componente di $\tilde{\sigma}_{i 1}(a)+\ldots+\tilde{\sigma}_{i s_{i}}(a)$ è elemento di $H$. Ne segue che $\xi_{i}=\tilde{\sigma}_{i 1}+\ldots+\tilde{\sigma}_{i s_{i}} \in \Pi_{i k}$ (veet $F$, vect $H$ ).

L'indipendenza di $\xi_{1}, \ldots, \xi_{t}$ su $\boldsymbol{Z}_{p}$, tenuto conto che $\left\{\tilde{\sigma}_{11}, \ldots, \tilde{\sigma}_{1 s_{1}}\right\}, \ldots,\left\{\tilde{\sigma}_{t 1}, \ldots, \tilde{\sigma}_{t s_{t}}\right\}$ è una partizione di $\left\{\tilde{\sigma}_{1}, \ldots, \tilde{\sigma}_{s}\right\}$, è conseguenza di 1.4 .

Che $\xi_{1}, \ldots, \xi_{t}$ generino $\Pi_{k}$ (vect $F$, vect $H$ ) su $\boldsymbol{Z}_{p}$, si prova con considerazioni sostanzialmente identiche a quelle che provano (cfr. 3.1) che $\eta_{1}, \ldots, \eta_{t}$ generano $\Pi_{k}(F, H)$ su $\boldsymbol{F}_{p}, \quad$ C.V.D.

\subsection{Corollario. - Sono equivatenti gli asserti:}

i) vect $F$ e vect $H$ sono isomorfi come $T_{k}$-moduli canonici,

ii) $F$ ed $H$ sono $\pi$-isomorfi come prolungamenti $d i k$.

Drm. - Che i) implichi ii), è banale. Viceversa, sia $f: F^{\prime} \rightarrow H$ un $\pi$-isomorfismo surgettivo; per 3.1 esistono $a_{1}, \ldots, a_{t} \in \boldsymbol{F}_{\triangleright}$ tali che

$$
f=a_{1}\left(\sigma_{11}+\ldots+\sigma_{1 s_{1}}\right)+\ldots+a_{t}\left(\sigma_{t 1}+\ldots+\sigma_{t s_{t}}\right) .
$$

Per 3.2, l'applicazione $g=\left\{a_{1}\right\}\left(\tilde{\sigma}_{11}+\ldots+\tilde{\sigma}_{1 s_{1}}\right)+\ldots+\left\{a_{t}\right\}\left(\tilde{\sigma}_{t 1}+\ldots+\tilde{\sigma}_{t s_{t}}\right)$ risulta un omomorfismo di vect $F$ in vect $H$ come $T_{k}$-moduli canonici. Tenuto conto che il $\pi$-omomorfismo di $F$ in $H$ indotto da $g$, è $f$ stesso, ed è pertanto un $\pi$-isomorfismo, si prova facilmente che $g$ è un isomorfismo surgettivo, C.V.D.

3.4. Teorkma. - Siano (cfr. [1]) $R_{F}$ ed $R_{H}$ le iperalgebre locali associate ad $F^{\prime}$ ed $H$; sono equivalenti gli asserti:

i) $R_{F} \cong R_{H}$,

ii) $F$ ed $H$ sono $\pi$-isomorfi come prolungamenti $d i k$.

Drm. - Siano $D_{F}, D_{H}$ le iperalgebre duali di $R_{F}, R_{H}$. Tenuto conto di 1.17 di [1] si ha $\checkmark D_{F} \cong$ vect $F, \cup D_{H} \cong$ vect $H$; ciò posto, l'asserto è consegúenza di 3.3. $\quad$ C.V.D.

4. - Siano $F, H$ prolungamenti finiti e separabili di $k$, e sia $N$ un prolungamento di Galois di $k$, contenente $F$ ed $H$.

Si ponga $G=\mathfrak{G}(N / k), \mathscr{F}=\mathfrak{G}(N / G), \mathscr{H}=\mathscr{S}(N / H)$.

Si considerino (cfr, 2) le algebre gruppali $\boldsymbol{F}_{p}[G], \boldsymbol{Z}_{p}[G]$.

Due ideali principali destri $a \cdot \boldsymbol{F}_{p}[Q], b \cdot \boldsymbol{F}_{p}[Q]$ di $\boldsymbol{F}_{p}[G]$, si diranno omotetici, se esiste $\xi \in \boldsymbol{F}_{p}[G]$ tale che l'applicazione $x \rightarrow \xi x$, ristretta ad $a \cdot \boldsymbol{F}_{p}[G]$, risulti un isomorfismo (di $\boldsymbol{F}_{p}[Q]$-moduli destri) di $a \cdot \boldsymbol{F}_{p}[G]$ su tutto $b \cdot \boldsymbol{F}_{p}[G]$.

Definizione analoga si dà per ideali principali destri di $\boldsymbol{Z}_{p}[G]$.

Si ponga infine: $\varrho_{\mathscr{F}}=\sum_{\sigma \in \mathcal{F}} \sigma$, $\varrho_{\mathcal{F}}=\sum_{\sigma \in \mathcal{F}} \sigma$, da interpretarsi, a seconda del contesto, come elementi di $\boldsymbol{F}_{p}[G]$, o di $\boldsymbol{Z}_{p}[G]$. 
4.1. Teorema. - Sono equivalenti gli asserti:

i) $F$ ed $H$ sono $\pi$-isomorfi come prolungamenti $d i k$,

ii) $\varrho_{\mathfrak{F}} \cdot \boldsymbol{F}_{p}[G]$ e $\varrho_{\mathfrak{H e}} \cdot \boldsymbol{F}_{p}[G]$ sono omotetici in $\boldsymbol{F}_{p}[G]$,

iii) $\varrho_{\mathscr{\mathcal { F }}} \cdot \boldsymbol{Z}_{y}[G]$ e $\varrho_{\mathcal{S}} \cdot \boldsymbol{Z}_{y}[G]$ sono omotetici in $\boldsymbol{Z}_{p}[G]$.

DrM. - Sia $\tilde{R}=\Pi_{k}\left(\right.$ vect $N$, vect $N$ ) (cfr. 2.2 ); in tale $Z_{x}$-algebra si ponga $\varrho_{\mathfrak{F}}=\sum_{\sigma \in \mathscr{F}} \tilde{\sigma}, \varrho_{\mathscr{X}}=\sum_{\sigma \in \mathfrak{X}} \tilde{\sigma}$.

Si osservi in primo luogo che $\varrho_{\mathscr{F}} \cdot \tilde{R}=\{f \in \tilde{R} / f($ vect $N) \subseteq$ vect $F\}$. Infatti: dato $f=\varrho_{\mathscr{F}} g$, per ogni $a \in$ vect $N$ ed ogni $\varrho \in \mathscr{F}$, si ha $\varrho f(a)=\left(\varrho_{\mathscr{F}}\right) g(a)=\varrho_{\mathscr{F}} g(a)=f(a)$; pertanto ogni componente di $f(a)$ è elemento di $F$; ne segue $f($ vect $N) \subseteq$ vect $F$. Viceversa, sia $f \in \tilde{R}$ tale che $f($ vect $N) \subseteq$ vect $F$; posto (cfr. 2.2) $f=\sum_{\sigma \in G} a_{\sigma} \tilde{\sigma}$, con gli $a_{\sigma} \in Z_{p}$ univocamente determinati, per ogni $\varrho \in \mathcal{F}$ si ha $\tilde{\varrho} f=f$, ossia $\sum_{\sigma \in G} a_{Q^{-1} \sigma} \cdot \tilde{\sigma}=\sum_{\sigma \in G} a_{\sigma} \tilde{\sigma}$; ne segue $a_{\varrho^{-1} \sigma}=a_{\sigma}$ per ogni $\sigma \in G$ ed ogni $\varrho \in \mathscr{F}$. Dette quindi $\mathscr{F}_{1}, \ldots, \mathscr{F} \varrho_{s}\left(s=\left[F^{*}: k\right]\right)$ le classi laterali destre di $\mathfrak{F}$ in $G$, si ha

$$
f=a_{\varrho_{1}}\left(\sum_{\sigma \in \mathscr{F}} \tilde{\sigma}\right) \tilde{\varrho}_{1}+\ldots+a_{\varrho_{s}}\left(\sum_{\sigma \in \mathcal{F}} \tilde{\sigma}\right) \tilde{\varrho}_{s}=\varrho_{\mathscr{F}}\left(a_{\varrho_{1}} \tilde{\varrho}_{1}+\ldots+a_{\varrho_{s}} \tilde{\tilde{e}}\right) \in \varrho_{\mathscr{F}} \cdot \tilde{R}
$$

Si noti in particolare che $\varrho_{\mathscr{F}} \cdot \tilde{R}$ risulta uno $\boldsymbol{Z}_{p}$-modulo libero di dimensione $[F: k]$, una cui base è $\varrho_{\mathscr{F}} \tilde{\varrho}_{1}, \ldots, \varrho_{\mathscr{F}} \tilde{\varrho}_{3}$.

Analogamente si prova che $\varrho_{\mathcal{F e}} \cdot \tilde{R}=\{f \in \tilde{R} / f($ vect $N) \subseteq$ vect $H\}$, e che $\varrho_{\mathcal{K}} \cdot \tilde{R}$ risulta uno $\boldsymbol{Z}_{p}$-modulo libero di dimensione $[H: k]$.

Si osservi in secondo luogo, che un elemento $f \in \tilde{R}$ è tale che $f($ veet $F) \subseteq$ vect $H$, se e solo se $f_{\mathscr{F}} \tilde{R} \subseteq \varrho_{\mathfrak{K}} \tilde{R}$. Infatti: le due precedenti osservazioni provano che, se $f($ vect $F) \subseteq$ vect $H$, risulta $f \varrho_{\mathscr{F}} \tilde{R} \subseteq \varrho_{\mathscr{H}} \tilde{R}$. Viceversa, essendo $f \varrho_{\mathscr{F}} \in \varrho_{\mathscr{H}} \widetilde{R}$, si ha intanto $f \varrho_{\mathscr{F}}($ vect $N) \subseteq$ vect $H$. Si consideri poi il $\pi$-omomorfismo di $N$ in $F$ indotto da $\varrho_{\mathfrak{F}}:$ vect $N \rightarrow$ vect $F$; tale $\pi$-omomorfismo risulta essere la traccia, $T_{N / F}$, da $N$ ad $F$; essendo $N$ separabile su $F, T_{N / F}$ non è nulla, ed essendo $T_{N / F}$ una applicazione $F$-lineare, $T_{X / F}$ risulta surgettiva; ne segue che l'omomorfismo $\varrho_{\mathscr{F}}$ : vect $N \rightarrow$ vect $F^{T}$ di $T_{h}$-moduli canonici, è anch'esso surgettivo, ossia che $\varrho_{\mathscr{F}}($ vect $N)=$ vect $F$. L'ultima uguaglianza, tenuto conto che $\varrho_{\mathscr{F}}($ vect $N) \subseteq$ vect $H$, prova che $f($ vect $F) \subseteq$ vect $H$.

Supponiamo quindi che esista un isomorfismo surgettivo $f:$ vect $F \rightarrow$ vect $H$, come $T_{k}$-moduli canonici. Per 1.3 , esiste un elemento $f_{1} \in \tilde{R}$, la cui restrizione a vect $F$ risulta $f$, ed un elemento $f_{2} \in \tilde{R}$, la cui restrizione a vect $H$ risulta $f^{-1}$ : vect $H \rightarrow$ $\rightarrow$ vect $F$. Si consideri l'applicazione di $\varrho_{\mathscr{F}} \tilde{R}$ in $\varrho_{\mathfrak{H}} \tilde{R}$ definita da $\xi \rightarrow f_{\mathrm{x}} \xi$ (si noti che, per $\xi \in \varrho_{\mathscr{F}} \tilde{R}$, si ha $\operatorname{Tm} \xi \subseteq \operatorname{vect} F$, e che pertanto $f_{1} \xi($ vect $N) \subseteq f_{1}($ vect $F)=$ $=f($ vect $F)=$ vect $H)$; tale applicazione risulta un isomorfismo surgettivo di $\tilde{R}$-moduli destri. Infatti: se risultasse $f_{1} \xi_{1}=f_{1} \xi_{2}$, essendo $\operatorname{Im} \xi_{1}, \operatorname{Im} \xi_{2} \subseteq$ veet $F$, ed essendo $f_{1}$ iniettiva su vect $F$, risulterebbe anche $\xi_{1}=\xi_{2}$. Dato inoltre $\eta \in \varrho_{x} \tilde{R}$, si consideri $f_{2} \eta$; essendo $\operatorname{Im} \eta \subseteq$ vect $H$, si ha $f_{2} \eta \in \varrho_{\mathscr{F}} \widetilde{R}$; essendo la restrizione $d i$ 
$f_{1} f_{2}$ a vect $H$ l'applicazione identica di vect $H$, tenuto nuovamente conto che $\operatorname{Im} \eta \subseteq$ vect $H$, si ha $f_{1}\left(f_{2} \eta\right)=\eta$.

Supponiamo viceversa che esista un elemento $f \in \tilde{R}$, tale che l'applicazione $\xi \rightarrow f \xi$, ristretta a $\varrho_{\mathscr{F}} \widetilde{R}$, sia un isomorfismo di $\widetilde{R}$-moduli destri, e quindi di $Z_{p}$-moduli, di $\varrho_{\mathscr{F}} \tilde{R}$ su tutto $\varrho_{\mathfrak{H}} \tilde{R}$. Si noti in primo luogo, che sotto tali ipotesi, le dimensioni di $\varrho_{\mathscr{F}} \tilde{R}$ e di $\varrho_{\mathfrak{X}} \tilde{R}$ su $\boldsymbol{Z}_{p}$ coincidono, e che quindi, per quanto precedentemente stabilito, risulta $[F: k]=[H: k]$. Si consideri quindi $f$; essendo $f \varrho_{\mathscr{F}} \in \varrho_{\mathscr{J}} \tilde{R}$, si ha $f_{\mathscr{F}}($ vect $N) \subseteq$ vect $H$, ed essendo $\varrho_{\mathscr{F}}($ vect $N)=$ vect $F$, si ha $f($ vect $F) \subseteq$ vect $H$; la restrizione $f^{\prime}$ di $f$ a veet $F$ è quindi un omomorfismo di vect $F$ in vect $H$, come $T_{k}$-moduli canonici. Inoltre, per le ipotesi poste su $f$, esiste $\xi \in \varrho_{\mathscr{F}} \widetilde{R}$ tale che $f \xi=\varrho_{\mathscr{K}} ;$ allora $f \xi($ vect $N)=\varrho_{\mathscr{H}}($ vect $N)=$ vect $H$, ed essendo $\xi($ vect $N) \subseteq$ veet $F$, si ha $f($ vect $F) \supseteq$ vect $H$. Essendo $f($ veet $F) \subseteq$ vect $H$, quanto precede prova che $f^{\prime}:$ vect $F \rightarrow$ $\rightarrow$ vect $H$ è surgettiva. Essendo $[F: k]=[H: k]$, le dimensioni di vect $F$ e vect $H$, come $T_{k}$-moduli canonici, coincidono (cfr. 1.16 di [1]); la surgettività di $f^{\prime}$, ne comporta allora la iniettività.

Le considerazioni precedenti provano che vect $F$ e vect $H$ sono isomorfi come $T_{k}$-moduli canonici, se e solo se $\varrho_{\mathscr{F}} \widetilde{R}$ e $\varrho_{\mathcal{F}} \widetilde{R}$ sono omotetici, e quindi, tenuto conto di 2.2 , se e solo se $\varrho_{\mathscr{F}} \cdot Z_{p}[G]$ e $\varrho_{\mathscr{H}} \cdot Z_{p}[G]$ sono omotetici.

Con procedimento analogo, si prova che $F$ ed $H$ sono $\pi$-isomorfi come prolungamenti di $k$, se e solo se $\varrho_{\mathscr{F}} \cdot \boldsymbol{F}_{p}[G]$ e $\varrho_{\mathfrak{H}} \cdot \boldsymbol{F}_{p}[G]$ sono omotetici.

Le ultime due considerazioni, tenuto conto di 3.3 , provano il teorema. C.V.D.

Tenuto conto di 3.4 e di 4.1, restringendo l'indagine a prolungamenti separabili di $k$, la congettura avanzata in [1], che prolungamenti finiti di $k$ aventi iperalgebre locali associate isomorfe, siano isomorfi, risulta equivalente a ciascuna delle seguenti congetture:

congettura $a)$. Siano $F, H$ prolungamenti finiti separabili di $k$; se $F$ ed $H$ sono $\pi$-isomorfi su $k$, allora $F$ ed $H$ sono isomorfi come prolungamenti di $k$;

congettura $b$ ). Sia $G$ un gruppo finito, e ne siano $\mathscr{F}$, J sottogruppi; se gli ideali $\varrho_{\mathscr{F}} \cdot \boldsymbol{F}_{p}[G]$ e $\varrho_{\mathcal{H}} \cdot \boldsymbol{F}_{p}[G]$ sono omotetici in $\boldsymbol{F}_{p}[G]$, allora $\mathcal{F}$ ed $\mathcal{H}$ sono coniugati rispetto ad un automorfismo interno di $G$;

congettura $o$ ). Sia $G$ un gruppo finito, e ne siano $\mathscr{F}$, Je sottogruppi; se gli ideali $\varrho_{\mathscr{F}} \cdot \boldsymbol{Z}_{p}[G]$ e $\varrho_{\mathcal{H}} \cdot \boldsymbol{Z}_{p}[G]$ sono omotetici in $\boldsymbol{Z}_{p}[G]$, allora $\mathscr{F}$ ed $\mathcal{H}$ sono coniugati rispetto ad un automorfismo interno di $G$.

5. - Siano $F, H$ prolungamenti finiti e separabili di $k$, e sia $N$ un prolungamento di Galois di $k$, contenente $F$ ed $H$.

Si ponga $G=\mathscr{S}(N / k), \mathfrak{F}=\mathfrak{S}(N / F), \mathfrak{H}=\mathfrak{S}(N / H)$.

(Per quanto concerne gli asserti in soli termini di algebre gruppali, le considerazioni contenute in questo paragrafo sussistono inalterate, ove in luogo di essere assegnati $k, F, H, N$, siano assegnati un gruppo finito $G$ e due suoi sottogruppi $\mathcal{F}$ ed $J$. In tale caso, nelle dimostrazioni, $k$ ed $N$ vanno interpretati come una qualsiasi coppia, 
certamente esistente, di corpi di earatteristica $p$, con $N$ di Galois su $k$, tale che $\mathcal{G}(N / k) \cong G ; F$ ed $H$ vanno poi interpretati come i sottocorpi di $N$ di stabilità per $\mathscr{F}$ ed $\mathfrak{K}$.)

5.1. TeOREMa. - Se l'ordine di $\mathfrak{F}$ è un numero primo, risultano equivalenti gli asserti:

i) $\varrho_{\mathscr{F}} \cdot \boldsymbol{F}_{p}[G]$ e $\varrho_{\mathfrak{H}} \cdot \boldsymbol{F}_{p}[G]$ sono omotetici in $\boldsymbol{F}_{p}[G]$,

ii) $\mathcal{F}$ ed $\mathfrak{H}$ sono coniugati rispetto ad un automorfismo interno $d i G$.

Drm. - Ovviamente ii) implica i). Viceversa: $\varrho_{\mathscr{F}} \cdot \boldsymbol{F}_{x}[G]$ e $\varrho_{\mathfrak{K}} \cdot \boldsymbol{F}_{p}[G]$, come spazi vettoriali su $\boldsymbol{F}_{p}$ hanno dimensioni (efr. la dimostrazione di 4.1) rispettivamente $(G: \mathfrak{F}),(G: \mathcal{H})$; essendo ovviamente isomorfi come spazi vettoriale, si ha $(G: \mathcal{F})=$ $=(G: \mathscr{H})$, pertanto gli ordini di $\mathscr{F}$ ed $\mathscr{H}$ coincidono. Poniamo: $q=$ ordine di $\mathfrak{F}=$ ordine di $\mathscr{H} ; q$, per ipotesi è primo.

Si consideri $\Pi_{k}\left(F^{\prime}, W^{\prime}\right)$. Siano $\left\{\sigma_{11}^{\prime} \mathscr{F}, \ldots, \sigma_{1 r_{1}}^{\prime} \mathfrak{F}\right\}, \ldots,\left\{\sigma_{h 1}^{\prime} \mathscr{F}, \ldots, \sigma_{h r_{h}}^{\prime} \mathfrak{F}\right\}$ le orbite in cui l'insieme $\left\{\sigma_{1} \mathscr{F}, \ldots, \sigma_{s} \mathscr{F}\right\}$ delle classi laterali sinistre di $\mathfrak{F}$ in $G$, risulta ripartito, quando $\mathcal{F}$ vi opera a sinistra. Per $3.1, \Pi_{k}(F, F)$, come spazio vettoriale su $\boldsymbol{F}_{p}$, ha dimensioni $h$. Essendo primo l'ordine $q$ di $\mathscr{F}$, ciascuna di tali orbite consta o di 1 o di $q$ elementi; inoltre esistono certamente orbite con 1 elemento (ad esempio l'orbita di $\mathcal{F}$ stesso come classe laterale). Detto $\lambda \neq 0$, il numero delle orbite con 1 elemento, si ha $h=\lambda+(s-\lambda) / q$; ne segue che $\Pi_{k}(F, F)$ consta di $p^{\lambda+(s-\lambda) / q}$ elementi.

Tenuto conto di 4.1, esiste un $\pi$-isomorfismo $f: F \rightarrow H$, su $k$; al variare di $g$ tra $\mathrm{i}$ $p^{\lambda+(s-\lambda) / a}$ elementi di $\Pi_{k s}(F, F)$, il prodotto $f g$ descrive allora $p^{\lambda+\left(s^{-\lambda}\right) / 2}$ elementi di $\Pi_{k}(F, H)$.

Si consideri quindi $\Pi_{k:}(F, H)$. Siano $\left\{\sigma_{11} \mathscr{F}, \ldots, \sigma_{1 s_{1}} \mathscr{F}\right\}, \ldots,\left\{\sigma_{t 1} \mathscr{F}, \ldots, \sigma_{t s_{t}} \mathcal{F}\right\}$ le orbite in cui $\left\{\sigma_{1} \mathscr{F}, \ldots, \sigma_{s} \mathscr{F}\right\}$ risulta ripartito quando $\mathscr{H}$ vi opera a sinistra. Per 3.1, $\Pi_{k}(F, H)$, come spazio vettoriale su $\boldsymbol{F}_{p}$, ha dimensione $t$. Essendo primo l'ordine $q$ di Je, ciascuna di tali orbite consta o di 1 o di $q$ elementi. Se ciascuna orbita constasse di $q$ elementi, si avrebbe $t=s / q$; pertanto $\Pi_{k}(F, H)$ consterebbe di $p_{-}^{s / q}$ elementi. Si avrebbe allora $\lambda+(s-\lambda) / q \leqslant s / q$, ossia $\lambda-\lambda / q \leqslant 0$; assurdo, essendo $1 \leqslant \lambda, 2 \leqslant q$.

Esiste allora un $\sigma_{i} \tilde{F}$, la cui orbita consta di un solo elemento; per ogni $\varrho \in \mathcal{H}$ si ha allora $\varrho \sigma_{i} \mathcal{H}^{\mathbb{K}}=\sigma_{i} \mathfrak{F}, \varrho \sigma_{i} \in \sigma_{i} \mathfrak{F}, \varrho \in \sigma_{i} \mathfrak{F}_{i}^{-1}$; ne segue $\mathcal{H} \subseteq \sigma_{i} \mathcal{F}_{i}^{-1}$, e quindi $\mathscr{H}=\sigma_{i} \mathfrak{F} \sigma_{i}^{-1}$, avendo entrambi tali gruppi ordine $q$. $\quad$ O.V.D.

5.2. Corollario. - Se $[N: F]$ è primo, $i$ prolungamenti di $k$ che risultano $\pi$-isomorfi ad $F$ su $k$, sono $i$ coniugati $d i F$ su $k$.

DIm. - Che i coniugati di $F$ su $k$ siano $\pi$-isomorfi ad $F$, è banale. Sia viceversa $K$ un prolungamento di $k, \pi$-isomorfo ad $F$; per 1.2 risulta $K \subseteq N$. Posto $\pi=\mathscr{G}(N / K)$, per 4.1, $\varrho_{\mathscr{T}} \cdot \boldsymbol{F}_{p}[G]$ e $\varrho_{\mathcal{H}} \cdot \boldsymbol{F}_{p}[G]$ sono omotetici in $\boldsymbol{F}_{p}[G]$. Essendo $[N: F]$ primo, per 5.1 esiste $\sigma \in G$ tale che $\pi=\sigma \mathscr{F} \sigma^{-1}$; ne segue $K=\sigma F$. O.V.D. 
5.3. TEOREMa. - Se l'ordine di $\mathfrak{F}$ è una potenza di p, risultano equivalenti gli asserti:

i) $\varrho_{\mathscr{F}} \cdot \boldsymbol{F}_{p}[G]$ e $\varrho_{\mathscr{H}} \cdot \boldsymbol{F}_{p}[G]$ sono omotetici in $\boldsymbol{F}_{p}[G]$,

ii) $\mathcal{F}$ ed $\mathcal{H}$ sono coniugati rispetto ad un automorfismo interno $d i G$.

DIM. - Ovviamente ii) implica i). Viceversa: siano $\left\{\sigma_{11} \mathfrak{F}, \ldots, \sigma_{1 s_{1}} \mathscr{F}\right\}, \ldots$, $\left\{\sigma_{t 1} \mathscr{F}, \ldots, \sigma_{t s_{s}} \mathscr{F}\right\}$ le orbite in cui l'insieme $\left\{\sigma_{1} \mathscr{F}, \ldots, \sigma_{s} \mathscr{F}\right\}$ delle classi laterali sinistre di $\mathfrak{F}$ in $G$, risulta ripartito quando $\mathfrak{T}$ si opera a sinistra.

Per 4.1, esiste un $\pi$-isomorfismo $f: F \rightarrow H$; fissato $f$, per 3.1 esistono e sono univocamente determinati $a_{1}, \ldots, a_{t} \in \boldsymbol{F}_{p}$ tali che la restrizione ad $F^{\prime}$ dell'elemento $f_{1}=a_{1}\left(\sigma_{11}+\ldots+\sigma_{1 s_{2}}\right)+\ldots+a_{t}\left(\sigma_{t 1}+\ldots+\sigma_{t s_{t}}\right)$ di $\Pi_{l i}(N, N)$, sia $f$. L'applicazione $\xi \rightarrow f_{1} \xi$, ristretta a $\varrho_{\mathscr{F}} \cdot \boldsymbol{F}_{p}[G]$, risulta (efr. la dim. di 4.1) un isomorfismo su tutto $\varrho_{y e} \cdot \boldsymbol{F}_{p}[G]$ (previa l'identificazione di $\Pi_{k}(N, N)$ con $\boldsymbol{F}_{p}[G]$; efr. 2.1).

In particolare esiste allora uno ed un solo $\xi \in \varrho_{\mathscr{F}} \cdot \boldsymbol{F}_{p}[G]$ tale che $f_{1} \xi=\varrho_{\mathfrak{H}}$. $\mathfrak{F}_{1}^{-1}, \ldots, \mathcal{F}_{\sigma_{s}}^{-1}$ sono le classi laterali destre di $\mathscr{F}$ in $G$; allora (cfr. la dim. di 4.1) $\varrho_{\mathscr{F}} \sigma_{1}^{-1}, \ldots, \varrho_{\mathscr{F}} \sigma_{s}^{-1}$ è una base di $\varrho_{\mathscr{F}} \cdot \boldsymbol{F}_{y}[G]$ su $\boldsymbol{F}_{p}$. Esistono quindi, e sono univocamente determinati, elementi $b_{1}^{\prime}, \ldots, b_{s}^{\prime} \in \boldsymbol{F}_{p}$, tali che $\xi=b_{1}^{\prime} \varrho_{\mathscr{F}} \sigma_{1}^{-1}+\ldots+b_{s}^{\prime} \varrho_{\mathscr{F}} \sigma_{s}^{-1}$, ossia tali che $f_{1} \cdot\left(b_{1}^{\prime} \varrho_{\mathscr{F}} \sigma_{1}^{-1}+\ldots+b_{s}^{\prime} \varrho_{\mathscr{F}} \sigma_{\mathrm{s}}^{-1}\right)=\varrho_{\mathscr{T}}$.

Si constata che $\left\{\mathscr{F} \sigma_{11}^{-1}, \ldots, \mathcal{F} \sigma_{1 s_{1}}^{-1}\right\}, \ldots,\left\{\mathscr{F} \sigma_{t 1}^{-1}, \ldots, \mathcal{F} \sigma_{t s_{t}}^{-1}\right\}$ sono le orbite in cui $\left\{\mathscr{F} \sigma_{1}^{-1}, \ldots, \mathscr{F} \sigma_{s}^{-1}\right\}$ risulta ripartito quando $\mathscr{H}$ si opera a destra. Fissato allora $i=1, \ldots, t$, e scelti $j, h$ compresi tra 1 ed $s_{i}$, esiste $\varrho \in \mathscr{H}$ tale che $\mathscr{F} \sigma_{i j}^{-1} \varrho=\mathscr{F} \sigma_{i \hbar}^{-1}$; per tale $\varrho$ si ha: $f_{1} \cdot\left(b_{1}^{\prime} \varrho_{\mathscr{F}} \sigma_{1}^{-1} \varrho+\ldots+b_{s}^{\prime} \varrho_{\mathscr{F}} \sigma_{s}^{-1} \varrho\right)=\varrho_{\mathfrak{H}} \varrho=\varrho_{\mathcal{H}}$. Essendo $\varrho_{\mathscr{F}} \sigma_{1}^{-1} \varrho, \ldots$, $\varrho_{\mathscr{F}} \sigma_{s}^{-1} \varrho$ una permutazione di $\varrho_{\mathscr{F}} \sigma_{1}^{-1}, \ldots, \varrho_{\mathscr{F}} \sigma_{s}^{-1}$, i coefficienti con i quali compaiono $\varrho_{\mathscr{F}} \sigma_{i \hbar}^{-1}$ e $\varrho_{\mathscr{F}} \sigma_{i t}^{-1}$ in $\xi$, coincidono. Detto quindi $b_{i}$ il coefficiente con il quale figura $\varrho_{\overparen{F}} \sigma_{i 1}^{-1}$ in $\xi$, si ha:

$$
\xi=b_{1} \varrho_{\mathscr{F}}\left(\sigma_{11}^{-1}+\ldots+\sigma_{1 s_{1}}^{-1}\right)+\ldots+b_{t} \varrho_{\mathscr{F}}\left(\sigma_{t 1}^{-1}+\ldots+\sigma_{t s_{t}}^{-1}\right) .
$$

Si ha allora:

$$
\begin{aligned}
& {\left[a_{1}\left(\sigma_{11}+\ldots+\sigma_{1 s_{1}}\right)+\ldots+a_{t}\left(\sigma_{t 1}+\ldots+\sigma_{t s_{2}}\right)\right] .} \\
& \qquad \varrho_{\mathscr{F}}\left[b_{1}\left(\sigma_{11}^{-1}+\ldots+\sigma_{1 s_{1}}^{-1}\right)+\ldots+b_{t}\left(\sigma_{t 1}^{-1}+\ldots+\sigma_{t s_{t}}^{-1}\right)\right]=\varrho_{\mathscr{H}} \cdot
\end{aligned}
$$

Esprimendo primo e secondo membro di tale uguaglianza come combinazioni lineari degli elementi di $G$, il confronto tra i coefficienti con i quali vi compare l'elemento neutro di $G$, prova che: $s_{1} a_{1} b_{1}+\ldots+s_{t} a_{t} b_{t}=1$.

Per l'ipotesi, si constata che l'ordine di $\mathfrak{H}$ coincide con quello di $\mathfrak{T}$, ed è pertanto una potenza di $p$; conseguentemente, $s_{1}, \ldots, s_{t}$, in quanto cardinalità di orbite di un insieme su cui opera $\mathfrak{H}$, sono potenze di $p$. Posto $s_{i}=p^{e_{i}}$, se per ogni $i$ fosse $e_{i} \neq 0$, risulterebbe: $s_{1} a_{1} b_{1}+\ldots+s_{t} a_{t} b_{t}=0$; assurdo.

Esiste pertanto una classe $\sigma_{i} \mathfrak{F}$, la cui orbita tramite $\mathfrak{K}$, consta di un solo elemento; come visto nelle considerazioni finali di 5.1, si ha allora $\mathcal{H}=\sigma_{i} \mathscr{F} \sigma_{i}^{-1}$. C.V.D. 
Ne seguono facilmente gli asserti:

5.4. CoROLLARIo. - Se $[N: F]$ è una potenza di $p$, i prolungamenti di k che risultano $\pi$-isomorfi ad $F$ su $k$, sono $i$ coniugati $d i F$ su $k$.

5.5. CoRollario. - Se G è un p-gruppo, sono equivalenti git asserti:

i) $\varrho_{\mathscr{F}} \cdot \boldsymbol{F}_{p}[G]$ e $\varrho_{\mathfrak{H}} \cdot \boldsymbol{F}_{p}[G]$ sono omotetici in $\boldsymbol{F}_{p}[G]$,

ii) $\mathcal{F}$ ed $\mathfrak{H}$ sono coniugati rispetto ad un automorfismo interno di $G$.

5.6. CoRollario, - $\$ e \mathfrak{G}(N / k)$ è un p-gruppo, sono equivalenti gli asserti:

i) $F$ ed $H$ sone $\pi$-isomorfi $s u k$,

ii) $F$ ed $H$ sono isomorfi come prolungamenti $d i k$.

\section{BIBLIOGRAFIA}

[MC] I. BarsotTI, Moduli eanonici e gruppi analitici commutativi, Ann. Scuola Norm. Sup. 13 (1959), pag. 303.

[MA] I. BARSOTTI, Metodi analitici per varietà abeliane in caratteristica positiva, Ann. Scuola Norm. Sup., capp. 1, 2, 14 (1964), pag. 1.

[1] M. PoLeTti, Prolungamenti finiti di un corpo e iperalgebre, Ist. Naz. Alta Mat., Symposia Math., 15 (1975), pag. 461.

[2] M. PoLETTI, Q-algebre p-adiche e loro rappresentazioni, Ann. Scuola Norm. Sup., 26 (1972), pag. 269.

[3] N. JACOBSON, Lectures in Abstract Algebra, vol. III, Van Nostrand, 1964. 